\section{Neuroblastic tumors and the epigenome}

In an attempt to discover new prognostic markers in patients with neuroblastic tumors, Banelli et al. have studied the CpG methylation patterns of a panel of genes thought to be involved in cancer development or drug responsiveness. The results of the study indicate that several genes bear distinct epigenetic signatures in different clinical groups of these tumors.

Using methylation-specific polymerase chain reaction or bisulfite restriction enzyme analysis, the investigators looked for distinct methylation profiles within the promoter regions of 11 genes. The tumor samples included 31 malignant neuroblastomas - of which 14 were MYCN-amplified and 17 were single-copy tumors-and 13 benign ganglioneuromas.

Of the 11 genes analyzed, five showed distinct methylation signatures in malignant and benign neuroblastic tumors. These were CASP8, 14.3.3 $\sigma, \triangle N p 73, R A S S F 1 A$, and DCR2. For 14.3.3 $\sigma, R A S S F 1 A$, and an intragenic segment of $C A S P 8$, different methylation patterns were found in the MYCN-amplified samples compared with the single-copy tumors, indicating that the methylation profile at these loci is associated with tumor aggressiveness.

The authors propose, therefore, that specific methylation profiles in patients with neuroblastic tumors might help in assessment of the risk of tumor progression. In addition, this approach might help in identifying those patients likely to benefit from particular therapies, as already demonstrated in colorectal cancer patients.

Ruth Kirby

Original article Banelli B et al. (2005) Distinct CpG methylation profiles characterize different clinical groups of neuroblastic tumors. Oncogene [doi: 10.1038/sj.onc.1208722]

\section{Measuring carotid plaque volume to evaluate treatments for atherosclerosis}

Use of 3D ultrasound to measure the volume of carotid plaque could be suitable for evaluating new treatments for atherosclerosis, an initial trial with atorvastatin has shown.

Previous studies carried out at the Robarts Research Institute in Canada have shown that carotid plaque area is a strong predictor of future cardiovascular events, including myocardial infarction and stroke.

In their recent study, Ainsworth et al. investigated whether transcutaneous carotid 3D ultrasound could detect the effects of atorvastatin treatment on the progression of atherosclerosis. Thirty-eight patients with carotid stenosis participated in this double-blind trial, and were randomly assigned to receive either $80 \mathrm{mg}$ atorvastatin daily $(n=17)$ or placebo $(n=21)$. Ultrasound scans of patients' carotid arteries were carried out at the start of treatment and at 3-month follow-up visits, with plaque volumes calculated using a 3D Quantify software program. Over 3 months, carotid plaque volume significantly decreased in patients receiving atorvastatin compared with those receiving placebo, showing that this technique could be used to evaluate antiatherosclerotic therapies.

Studies involving alternative methods for assessing the degree of atherosclerosis, for example the measurement of coronary calcification and carotid intima-media thickness, are limited by long treatment regimens and the need for large sample sizes. The small number of patients in this study and its relatively short duration highlight potential advantages of measuring the volume of carotid plaque in future trials evaluating new treatments.

Claire Braybrook

Original article Ainsworth CD et al. (2005) 3D ultrasound measurement of change in carotid plaque volume. A tool for rapid evaluation of new therapies. Stroke [doi: 10.1161/ 01.STR.0000178543.19433.20]

\section{Artificial disc proves a success in prospective, randomized trial}

Promising results were recently reported from a prospective, randomized study of the CHARITÉ ${ }^{\circledR}$ artificial disc (DePuy Spine, Raynham, MA), a device created for the surgical treatment of degenerative disc disease (DDD). Currently, DDD is surgically treated with lumbar fusion.

Blumenthal et al. enrolled 304 patients with DDD (spinal levels L4-S1) at 14 centers throughout the US. Patients were randomized to treatment with the artificial disc or anterior lumbar interbody fusion (controls) in a 2:1 ratio. A clinical assessment was undertaken before surgery, with further assessment at 6 weeks, and 3, 6, 12 and 24 months post-surgery. Clinical outcomes were assessed using questionnaires such as the 